\title{
The application of SiPMs in the fluorescence telescope FAMOUS and the Aachen Muon Detector
}

\author{
J. Kemp*, T. Bretz, T. Hebbeker, L. Middendorf, T. Niggemann, C. Peters and \\ J. Schumacher \\ III. Physikalisches Institut A \\ RWTH Aachen University, Germany \\ E-mail: kemp@physik.rwth-aachen.de
}

\begin{abstract}
After huge advancements in SiPM technology made in the last years, they are perfect sensors for light detection in astroparticle physics experiments. They are very robust devices and have an equal or higher photon detection efficiency than conventional photomultiplier tubes (PMTs). In addition, SiPMs can be precisely calibrated exploiting their single photon resolution. We study their performance in various applications.

FAMOUS (First Auger Multi-pixel photon counter camera for the Observation of Ultra-highenergy air Showers) is a fluorescence telescope with a 61-pixel camera made of SiPMs. It is a small sized telescope using a Fresnel lens as the focusing element.

The Aachen Muon Detector (AMD) is a scintillator detector designed to improve current experiments through a precise determination of the muon content in air-showers. The light produced in scintillating tiles is collected by wavelength-shifting fibers. Through clear fibers the light is guided on one SiPM per tile.
\end{abstract}

35th International Cosmic Ray Conference

10-20 July, 2017

Bexco, Busan, Korea

\footnotetext{
* Speaker.
} 


\section{Introduction}

Current ground-based astroparticle physics experiments observe extensive air-showers evolving in the Earth's atmosphere. Their evolution can be observed using telescopes detecting the Cherenkov light (e.g. VERITAS [1]) or fluorescence light (e.g. the Pierre Auger Observatory [2]) produced by charged particles passing through the atmosphere. However, these techniques only allow for an observation during clear moonless nights. A higher duty cycle is achieved with particle detectors measuring only the shower footprint at ground. This can be done by placing tanks filled with water (e.g. Pierre Auger Observatory [3], HAWC [4]) or ice (e.g. IceTop [5]) at the Earth's surface or using scintillator based detectors (e.g. Telescope Array [6]).

All of these techniques have in common that very low light fluxes have to be detected with good timing resolution. Thus, for several decades the only device in question to be used in such detectors was the photomultiplier tube (PMT) which is capable of detecting single photons. PMTs have already been developed in the early $20^{\text {th }}$ century [7]. Since then, they have improved significantly and they have been the major photon detector used in astroparticle physics experiments when a high gain and sensitivity is needed. Nonetheless, the semiconductor industry has also made huge progress in the last decades allowing for sensitive and precise semiconductor based photosensors. These include sensors typically used in digital photo cameras like CCD- or CMOS-sensors, but also sensors suitable for detecting lower light fluxes such as avalanche photo-diodes (APDs). When operating APDs in Geiger mode (G-APD) above their breakdown voltage they allow for single-photon detection with high sensitivity. A silicon photomultiplier (SiPM) is compiled of several thousand G-APDs. Compared to PMTs they have a similar or higher photon detection efficiency and do not age when exposed to bright light. The latter allows telescopes to perform observations even during full moon.

These properties make SiPMs ideally suited for astroparticle physics experiments.

\section{Silicon Photomultipliers}

SiPMs are compiled of several thousands of G-APD cells. Thus, SiPMs are operated at a voltage $V_{o p}$ which is higher than the breakdown voltage $V_{b r}$ of each cell. The breakdown voltage is typically below $100 \mathrm{~V}$ and around $50-60 \mathrm{~V}$ for recent Hamamatsu devices. The difference $V_{o p}-V_{b r}=V_{o v}$ is called the overvoltage. It is usually in the order of a few volts. The gain depends linearly on the overvoltage and is in the order of $10^{6}$ [8]. In addition, the photon detection efficiency (PDE) and the dark noise increase with overvoltage. Thus, for each application a compromise between gain, PDE and noise has to be found to choose an optimal overvoltage.

The breakdown voltage of SiPMs depends on the temperature. For recent Hamamatsu devices it is around $\sim 60 \mathrm{mV} / \mathrm{K}$. To keep PDE and gain stable, the operating voltage has to be adjusted accordingly when the SiPM is operated in an environment with varying temperature. Thus, a temperature sensor and an active adjustment of the operating voltage have to be implemented.

The main contribution to the dark noise of an SiPM is thermal noise caused by thermal excitations in a cell. In addition, 'optical crosstalk' is induced by photons that are produced during a cell breakdown and trigger neighboring cells. For older SiPMs this effect occurs in $>20 \%$ [9] of the cell breakdowns but it is reduced to only a few percent for recent devices [10]. 'Afterpulsing' 
results from impurities in the silicon lattice structure trapping electrons or holes of the avalanche. They are released with a delay of some ten nanoseconds and induce a new avalanche. This effect is negligible in recent devices. As crosstalk and afterpulsing are always induced by a cell breakdown they are called 'correlated noise' while thermal noise is referred to as 'uncorrelated noise'.

\section{The Aachen Muon Detector}

\subsection{Motivation}

Current air-shower detectors using telescopes or water-Cherenkov detectors (WCD) on ground are not able to discriminate between the electromagnetic and muonic shower component. However, the muon-to-electron ratio is a key quantity to determine the mass of the primary particle and thus to the precise measurement of the mass composition [11].

The Aachen Muon Detector (AMD) is a scintillator based detector designed to detect the muonic component of extensive air-showers.

\subsection{The detector design}

To detect mainly the muonic component of an air-shower, AMD has to be shielded from the electromagnetic shower component. The shield can be for example realized by a WCD as used at the Pierre Auger Observatory. AMD is placed under the WCD and a separation of the electromagnetic and muonic component is possible by comparing the signals of the two detectors. A sketch of the setup is shown in figure 1 .

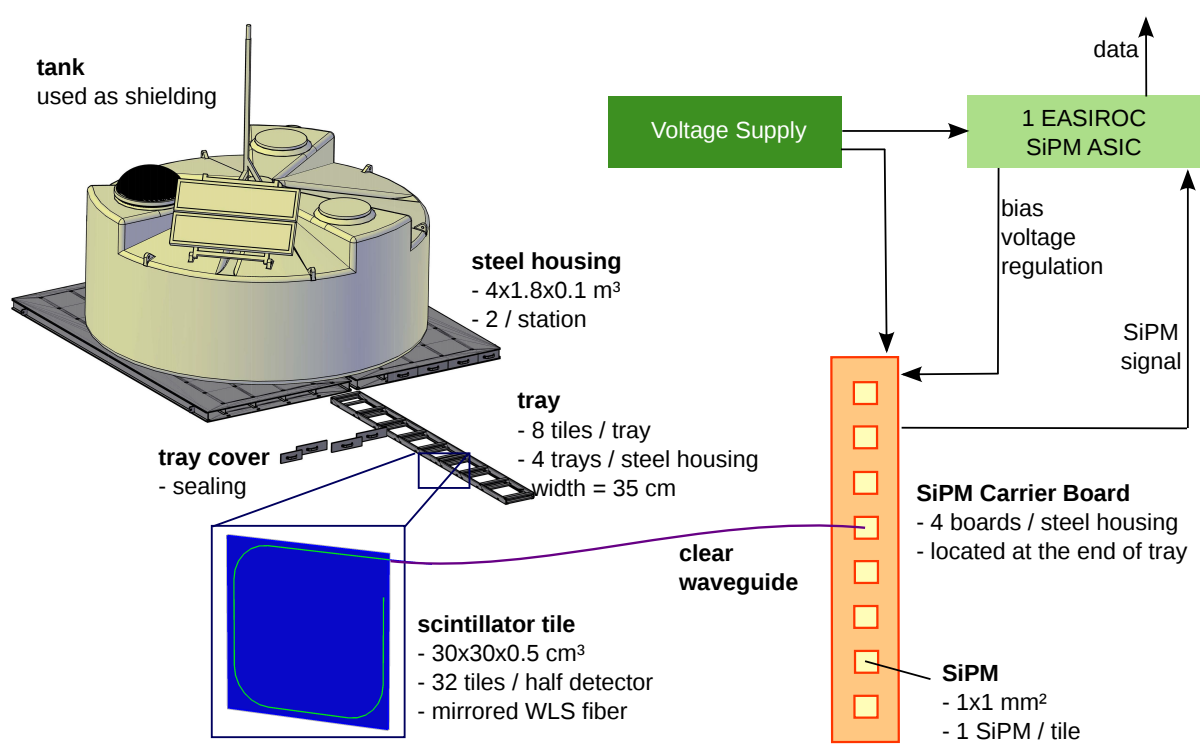

Figure 1: Sketch of the setup of the AMD. Two steel housings hosting the scintillator tiles are placed under one WCD. The light is guided on one SiPM per tile. Read out and temperature correction of the supply voltage is performed by the EASIROC.

To support a WCD, AMD has to be mechanically very robust. All the components are placed inside a steel housing with a size of approximately $4 \times 1.8 \times 0.1 \mathrm{~m}^{3}$. A detector station consists 
of two such steel housings. One detector station consists of 64 scintillator tiles (plastic scintillator EJ-212 from Eljen Technology [12]) sized $30 \times 30 \times 0.5 \mathrm{~cm}^{3}$. Eight each are lined up in eight trays with a distance of $7.5 \mathrm{~cm}$ between two tiles. The trays are placed in the two steel housings (fig. 2).

Light produced in the scintillator tiles by through-going charged particles is collected in a wavelength-shifting fiber (WLS). It is glued in a groove with a sigma shape in the tile (fig. 3).

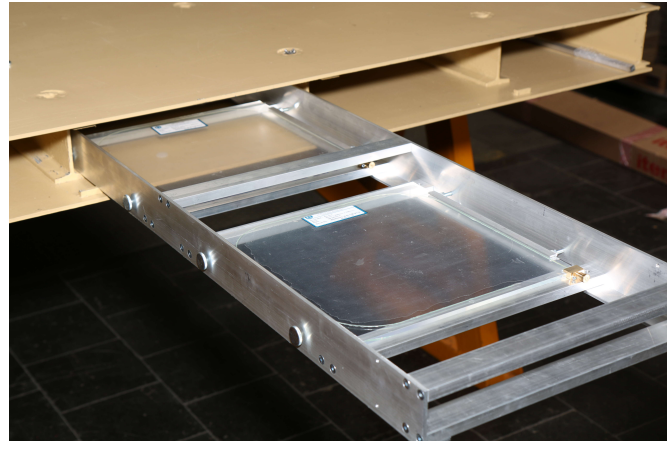

Figure 2: The tray holding the scintillator tiles.

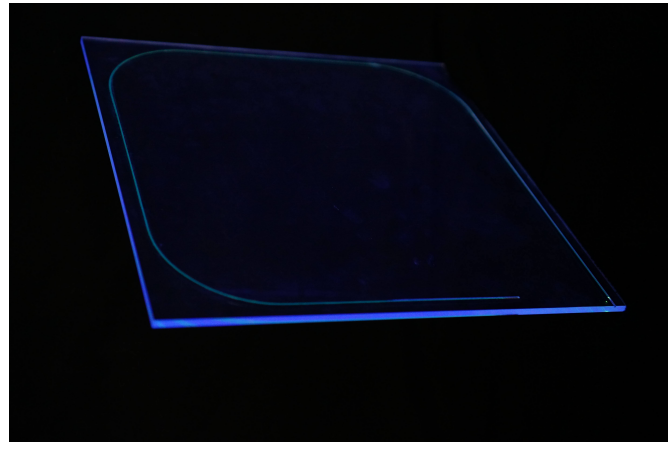

Figure 3: A scintillator tile with a glued WLS.

One fiber end is mirrored while the other end is guided to the edge of the tile. It is connected to a clear optical fiber. Due to the easy operation of SiPMs it is possible to use one SiPM per optical fiber. Eight SiPMs are carried by one SiPM board which is placed at the end of each tray. Thus, the fibers have lengths between $0.3 \mathrm{~m}$ and $2.9 \mathrm{~m}$.

The SiPMs used for AMD are sized $1 \times 1 \mathrm{~mm}^{2}$ with a cell pitch of $50 \mu \mathrm{m}$ (Hamamatsu S12571050P [9]). Each SiPM has a slightly different operation voltage in the range of $67 \pm 1 \mathrm{~V}$. Thus, the bias voltage has to be set for each SiPM individually and is automatically corrected according to the ambient temperature. The readout board is based on the EASIROC (Extended Analogue SI-pm ReadOut Chip). It is an ASIC developed for the usage with SiPMs by Omega Micro and offers 32 analogue front-end channels [13]. Each signal channel is processed by a fast shaper and followed by a discriminator that can be set with a 10-bit DAC. Using a slow shaper and external ADC, one signal integral can be recorded per event and channel.

The readout board is also equipped with an FPGA (Field Programmable Gate Array) that allows for communication with the EASIROC, communication with adjacent detectors (such as a WCD) and temporary data storage. As the EASIROC has only 32 channels, two such boards are used for one AMD station. More details on AMD can be found in [14].

\subsection{MiniAMD}

MiniAMD is a detector based on the knowledge gained during the development of AMD. Its design is supposed to allow for easy handling with only two persons. Thus, it is well suited for calibration and testing of larger detectors in the field such as the future Surface Scintillator Detector at the Pierre Auger Observatory [15]. MiniAMD is a smaller version of AMD with eight scintillator tiles placed in an aluminum box in two rows of four tiles. The size of the box will only be about $1.4 \times 0.8 \times 0.1 \mathrm{~m}^{3}$ with a weight of less than $20 \mathrm{~kg}$. A schematic is shown in figure 4 . For MiniAMD, the fibers will not be glued with the tile. Instead, a groove that is only slightly wider than the fiber is milled out of the tile. Thus, the fiber is only clamped mechanically in the groove. 


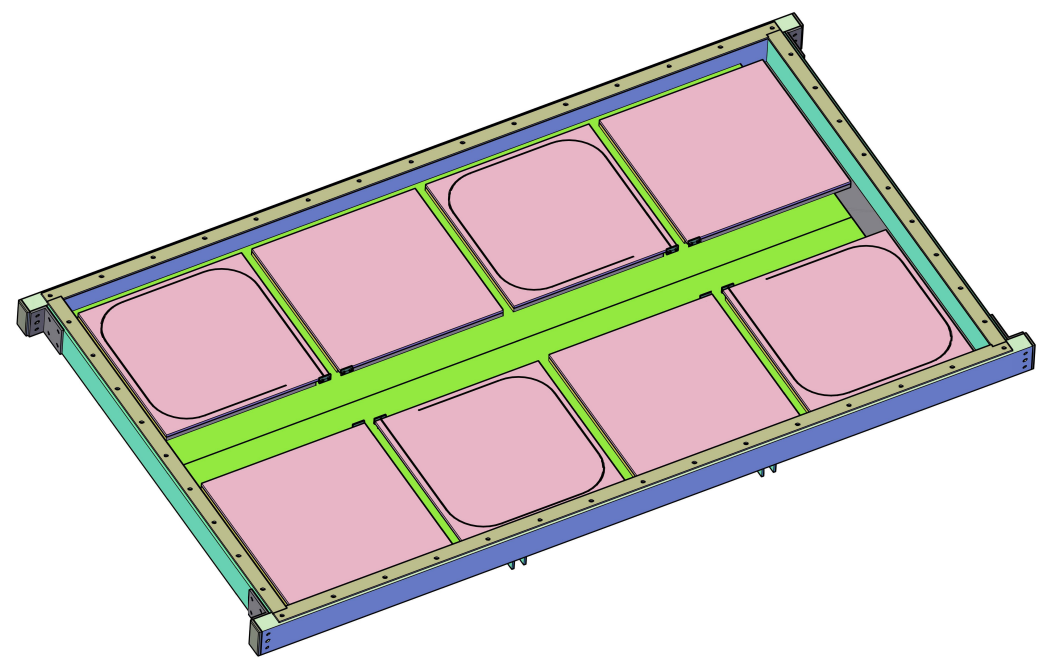

Figure 4: A schematic of the MiniAMD with only eight scintillator tiles.

Only the fiber ends are fixed with a small portion of glue to avoid that the fiber moves in the tile. This procedure is significantly simpler than gluing the whole fiber. The fiber end is flush with the edge of the tile.

In contrast to the AMD detector, in MiniAMD the SiPMs are mounted directly at the scintillator tiles. Thus, no coupling between a WLS and a clear optical fiber is needed reducing complexity and lightloss. The SiPMs used for MiniAMD are the latest types of SiPMs offered by Hamamatsu (S13360-1350PE [10]). They have a size of $1.3 \times 1.3 \mathrm{~mm}^{2}$ with a cell pitch of $50 \mu \mathrm{m}$. Especially the crosstalk probabilitiy has been reduced compared to older versions. The EASIROC board needed for readout and triggering will not be contained in the box. Instead, it is placed outside and can be connected to up to four MiniAMD detectors. This allows for easy trigger generation of multiple modules (e.g. one above, one below the detector under test) without the need for complex synchronization between the two.

A MiniAMD detector will be ready for measurements later in 2017.

\subsection{First measurements with a MiniAMD tile}

Before assembling the detector and finalizing the design, the performance of the components has to be studied. Thus, especially the amount of light detected for a minimum ionizing particle (MIP) passing through a scintillator tile has to be measured. It should be well above the noise to ensure that also triggering on single MIP is possible.

In order to measure the light output of a scintillator tile, a stack of three tiles was set up. A sketch is shown in figure 5. The top and bottom tiles are set in coincidence to trigger on muons and to suppress random noise. The tile in the middle is the one under study. The result is shown in figure 6. The MIP peak at around 2200 ADC counts is well above the background. In addition, the peaks corresponding to the detection of multiple photons are nicely separated. From simple counting one can estimate the amount of detected photons per MIP. For the tile used in MiniAMD it is around 23 photons per MIP. 


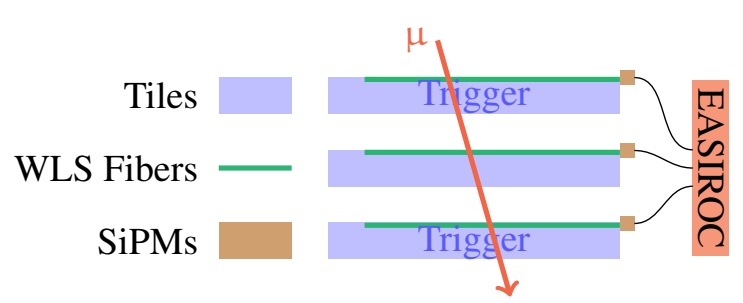

Figure 5: A sketch of the setup used to measure the light output of a scintillator tile. The setup consists of three tiles, two serving as a trigger and one to be measured.

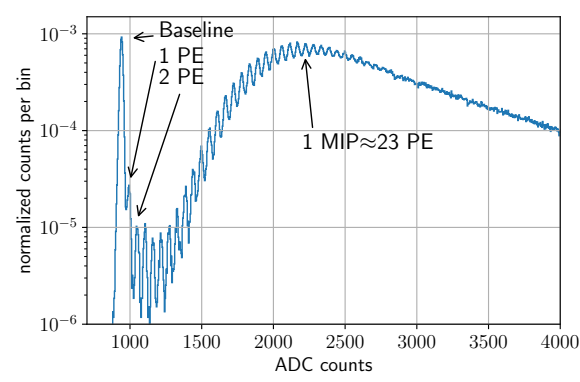

Figure 6: Result of the measurement of the light output of a scintillator tile. The MIP peak is nicely visible and the peaks corresponding to the detection of multiple photons are well separated.

\section{The Fluorescence Telescope FAMOUS}

\subsection{Motivation}

Current fluorescence telescopes like the ones at the Pierre Auger Observatory use PMTs to detect the fluorescence light produced by cosmic-ray air-showers in the atmosphere. The duty cycle of these telescopes is limited to dark nights due to the fast aging of PMTs when exposed to high light fluxes. Thus, operation is not possible during full moon nights or when the moon is directly shining into the telescope. In contrast, SiPMs are very robust and do not suffer from accelerated aging when exposed to high light fluxes. A telescope equipped with SiPMs can therefore also perform observations even when the moon is directly shining into the camera [16].

\subsection{The design of FAMOUS}

FAMOUS (First Auger Multi-pixel photon counter camera for the Observation of Ultra-highenergy air Showers) is designed as a proof-of-principle to demonstrate the advantages of SiPMs in a fluorescence telescope [17].

FAMOUS has a $50 \mathrm{~cm}$ diameter aluminum tube fixed on an altitude-azimuth mount. It is equipped with a Fresnel lens with a diameter of $50 \mathrm{~cm}$ and about the same focal length. Using a Fresnel lens allows for a light-weight design and for easy handling even with only one person. A schematic of the optical setup is shown in figure 7.

The light is focused on a 61-pixel camera. Three additional channels are blinded and slightly separated from the rest. They allow for monitoring the pure dark and electronics noise. Each pixel consists of a Winston cone guiding the light on an array of $2 \times 2$ SiPMs (Hamamatsu S10985-100C [18]). Each SiPM has a size of $3 \times 3 \mathrm{~mm}^{2}$ and a cell pitch of $100 \mu \mathrm{m}$. The large cell pitch allows for a large effective detection area and a high gain of the cell. The peak sensitivity is reached at a wavelength of $440 \mathrm{~nm}$. The usage of Winston cones increases the effective detection area and therefore the light yield of each pixel. These Winston cones are circular with an entrance radius of $6.71 \mathrm{~mm}$ and an exit radius of $3 \mathrm{~mm}$. The field of view of one pixel is about $1.5^{\circ} \times 1.5^{\circ}$.

The readout is performed for each pixel individually using a Target-7 chip [19]. One chip has 16 channels and thus 4 chips are used for the telescope. Digitization is performed at a sampling rate of $200 \mathrm{MS} / \mathrm{s}$ to $1.4 \mathrm{GS} / \mathrm{s}$ with a 10 bit resolution. The buffer length for each channel is 16,384 


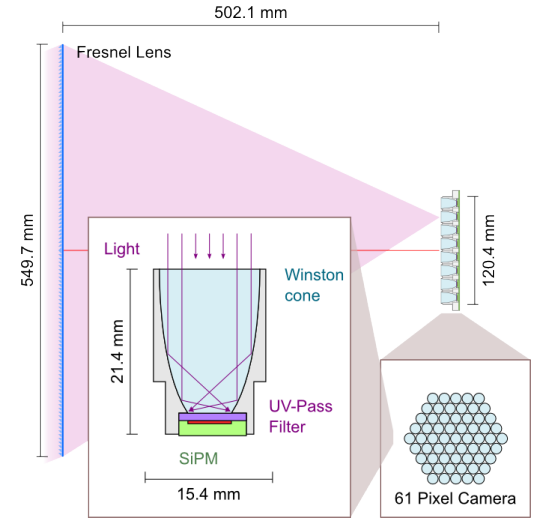

Figure 7: A sketch of the FAMOUS telescope. The light is focused by a Fresnel lens. Each pixel consists of a light guiding Winston cone screwed on the SiPM board.

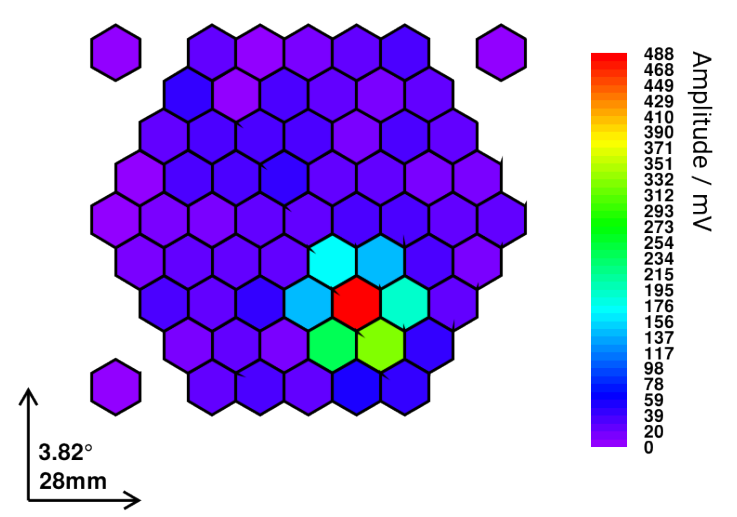

Figure 8: A cosmic-ray air-shower as seen with the FAMOUS telescope. Each hexagon corresponds to one pixel. Three pixels are separated from rest and are blinded. They allow for monitoring the noise.

samples allowing for the observation of signals with lengths at the order of $\sim 10 \mu \mathrm{s}$. This is an important feature, as typical signals from fluorescence light produced by air-showers can last several $\mu$ s. The power supply for the SiPMs is custom made and allows to set the bias voltage for each pixel separately including automatic correction according to the ambient temperature [20].

\subsection{FAMOUS at HAWC}

FAMOUS being a light-weight telescope makes it ideally suited to be used at remote places or as an extension to existing observatories. Thus, a telescope that will be brought to the site of the HAWC Observatory [4] in Mexico is currently in production and being tested [21].

For operation in coincidence with HAWC, FAMOUS is optimized for the observation of Cherenkov light instead of fluorescence light. Instead of using the Target-7 chip, the read-out is based on DRS4 chips [22] allowing for a sampling rate of up to $5 \mathrm{GS} / \mathrm{s}$.

Setting up FAMOUS at the HAWC site will allow for detailed studies of the FAMOUS performance by exploiting information provided by HAWC.

\subsection{First test measurement}

The FAMOUS telescope that will be operated at the HAWC site has already been set up and first test measurements have been performed. It has been operated outside observing cosmic-ray air-showers. In figure 8 an example of an event is shown. Each of the hexagons symbolizes one pixel and the color corresponds to the measured integrated signal.

\section{Summary \& Outlook}

The development of SiPMs in the recent years made them mature and ideally suited for applications in astroparticle physics experiments. They are very robust, have a high PDE and can be operated also at brighter conditions than classical PMTs. The development of AMD and FAMOUS exploits these advantages. 
MiniAMD will be set up by the end of the year 2017. Test measurements will be performed and it can be used as a testing and calibration device for other scintillator detectors. Its low weight and easy handling make it the perfect choice for such applications.

A FAMOUS telescope equipped with 61 pixels is already being tested in the lab. It is being characterized and its properties are under study. It is planned to bring one FAMOUS telescope to the HAWC Observatory in Mexico allowing for a precise characterization of the FAMOUS telescope.

Acknowledgements The work on AMD and FAMOUS was funded by the German Federal Ministry of Education and Research (BMBF) and the RWTH Aachen University. The Helmholtz Alliance for Astroparticle Physics also funded the AMD project. The Astroparticle Eranet also funded the FAMOUS project. We appreciated the support from the Pierre Auger Collaboration and from the local IceCube group. We thank the staff at the electronics and mechanics workshop in Aachen.

\section{References}

[1] F. Krennrich et al., New Astron. Rev. 48 (2004) 345-349.

[2] Pierre Auger Collaboration, J. Abraham et al., Nucl. Instrum. Meth. A620 (2010) 227-251.

[3] Pierre Auger Collaboration, I. Allekotte et al., Nucl. Instrum. Meth. A586 (2008) 409-420.

[4] HAWC Collaboration, A. U. Abeysekara et al., arXiv:1310.0074.

[5] IceCube Collaboration, R. Abbasi et al., Nucl. Instrum. Meth. A700 (2013) 188-220.

[6] T. Abu-Zayyad et al., Nuclear Instruments and Methods in Physics Research A 689 (Oct., 2012) 87-97.

[7] V. K. Zworykin and J. A. Rajchman, Proceedings of the IRE 27 (Sept, 1939) 558-566.

[8] D. Renker and E. Lorenz, JINST 4 (2009) P04004.

[9] Hamamatsu Corporation, Hamamatsu S12571-050 datasheet, June, 2017.

[10] Hamamatsu Corporation, Hamamatsu S13360 datasheet, June, 2017.

[11] K.-H. Kampert and M. Unger, Astropart. Phys. 35 (2012) 660-678.

[12] Eljen Technology, Eljen Technology EJ-212 Data Sheet, June, 2017.

[13] S. Callier, C. D. Taille, G. Martin-Chassard, and L. Raux, Physics Procedia 37 (2012) 1569 - 1576.

[14] C. Peters et al., PoS ICRC2015 (2015) 596.

[15] Pierre Auger Collaboration, A. Aab et al., arXiv:1604.03637.

[16] H. Anderhub et al., Journal of Instrumentation 8 (2013) P06008.

[17] T. Bretz et al., PoS ICRC2015 (2015) 606405.

[18] Hamamatsu Corporation, Hamamatsu S10985 datasheet, June, 2017.

[19] K. Bechtol, S. Funk, A. Okumura, L. Ruckman, A. Simons, H. Tajima, J. Vandenbroucke, and G. Varner, Astroparticle Physics 36 (2012) $156-165$.

[20] J. Schumacher et al., PoS ICRC2015 (2015) 605.

[21] M. Schaufel et al., PoS ICRC2017 (2017) 786.

[22] Paul Scherrer Institut, DRS 4 chip, June, 2017. 